\title{
Regulated provider perceptions of feedback reports
}

\author{
HANNAH M. O'ROURKE RN BScN (Hons) ${ }^{1}$, KIMBERLY D. FRASER RN PhD ${ }^{2}$, ANNE-MARIE \\ BOSTRÖM RN PhD ${ }^{3,4,5}$, MELBA ANDREA B. BAYLON MSPH ${ }^{6}$ and ANNE E. SALES RN PhD ${ }^{7,8}$ \\ ${ }^{1} \mathrm{PhD}$ Student ${ }^{2}$ Assistant Professor, Faculty of Nursing, University of Alberta, Edmonton, Alberta, Canada, \\ ${ }^{3}$ Associate Professor, Department of Neurobiology, Care Science and Society, Division of Nursing, Karolinska \\ Institutet, Huddinge, ${ }^{4}$ Associate Professor, Department of Geriatric Medicine, Danderyd Hospital, Danderyd, \\ Sweden, ${ }^{5}$ Assistant Adjunct Professor, ${ }^{6}$ Statistician Faculty of Nursing, University of Alberta, Edmonton, Alberta, \\ Canada, ${ }^{7}$ Professor, School of Nursing, University of Michigan, Ann Arbor, MI and ${ }^{8}$ Research Scientist, Center \\ for Clinical Management Research, VA Ann Arbor Healthcare System, Ann Arbor, MI, USA
}

Correspondence

Hannah M. O'Rourke

clo Graduate Programs

Faculty of Nursing

4-171 Edmonton Clinic Health

Academy

1140587 Avenue

University of Alberta

Edmonton

Alberta T6G 1C9

Canada

E-mail: hannah.orourke@ualberta.ca
O'ROURKE H.M., FRASER K.D., BOSTRÖM A.-M., BAYLON M.A.B. \& SALES A.E. (2013) Journal of Nursing Management 21, 1016-1025.

\section{Regulated provider perceptions of feedback reports}

Aim This paper reports on regulated (or licensed) care providers' understanding and perceptions of feedback reports in a sample of Canadian long-term care settings using a cross-sectional survey design.

Background Audit with feedback quality improvement studies have seldom targeted front-line providers in long-term care to receive feedback information. Methods Feedback reports were delivered to front-line regulated care providers in four long-term care facilities for 13 months in 2009-10. Providers completed a postfeedback survey.

Results Most $(78 \%)$ regulated care providers $(n=126)$ understood the reports and felt they provided useful information for making changes to resident care $(64 \%)$. Perceptions of the report differed, depending on the role of the regulated care provider. In multivariable logistic regression, the regulated nurses' understanding of more than half the report was negatively associated with 'usefulness of information for changing resident care', and perceiving the report as generally useful had a positive association.

Conclusions Front-line regulated providers are an appropriate target for feedback reports in long-term care.

Implications for nursing management Long-term care administrators should share unit-level information on care quality with unit-level managers and other professional front-line direct care providers.

Keywords: audit, feedback, long-term care, quality improvement

Accepted for publication: 18 January 2013

\section{Introduction}

Demographic trends in Canada indicate that the proportions of Canadians over the ages of 65 and 85 are increasing, and will continue to do so at accelerating rates over the next several decades (Statistics Canada
2008, McDaniel 2009). While many older adults remain independent, the risk of having one or more chronic diseases and of requiring supportive care increases with age. Even though only $2 \%$ of those aged $65-74$ receive care and live in a long-term care (LTC) facility, this number increases to $32 \%$ for those aged 85 years and 
older (Statistics Canada 2007). Furthermore, in recent years, residents in LTC facilities have displayed higher levels of acuity, complexity, and functional care needs than in the past (Alberta Office of the Auditor General 2005) and staff struggle as their roles expand to meet the needs of residents and their families (McGilton et al. 2007). Regulated nurses (in Canada, these are nurses who obtain licensure through a regulatory body) comprised just $27 \%$ of providers in LTC in 2007; unregulated care providers (or health care aides) delivered $72 \%$ of direct care (Report of the Auditor General of Alberta 2008). The presence of regulated allied health professionals (AHPs) (pharmacists, social workers and rehabilitation professionals) varies widely in LTC settings.

\section{Attempts to improve care quality in long-term care settings}

Since the 1980s, groups have advocated improving the care given to older adults with complex health care needs in long-term care (LTC) environments. The Institute of Medicine's influential report highlighted the high prevalence of poor care practices in LTC facilities in the United States (Institute of Medicine 1986). This led to the 1987 Omnibus Budget Reconciliation Act (OBRA), which put forward new nursing home standards and inspection and enforcement processes.

Another important change resulting from the OBRA was the development and implementation of a standardized system for assessment and documentation for individuals admitted to LTC facilities: the Resident Assessment Instrument-Minimum Data Set (RAI) (Hawes et al. 1995). In addition to care planning, the RAI data have been used as a data source to derive measures of care quality (quality indicators), which can be used to compare and benchmark LTC facilities on important areas relevant to the quality of care that residents receive (such as pain, falls and depression) (Zimmerman 2003).

In Canada, the debate surrounding the quality of LTC services gained momentum more recently (Berta et al. 2006). In Alberta, Canada, many activities have been undertaken to resolve quality of care problems. These include increasing external oversight to ensure care facilities meet basic standards of care and implement the RAI, develop resident care plans and measure quality of care (Institute of Medicine 2001, Alberta Office of the Auditor General 2005, Saher 2011). Similar recommendations to improve quality have been made in other Canadian provinces (Hirdes et al. 2011).

Despite these efforts and the evidence of improvements in some areas of care, concerns about quality persist (Institute of Medicine 2001, Saher 2011). There are many suggested strategies for improving care, including strengthening regulatory processes, enhancing the caregiver workforce, improving data used for quality monitoring and changing the nursing home culture (Wiener 2003). However, a lack of evidence supporting the feasibility and efficacy of these strategies is a barrier to their implementation. There is a need to establish which quality improvement interventions are both feasible and effective within the LTC context (Wiener 2003, Shojania \& Grimshaw 2005, Berwick 2008, Swafford et al. 2009, Bostrom et al. 2012). To date, literature reviews of quality improvement research highlight that there has been a lack of quality improvement research conducted specifically in LTC settings compared with other health care settings (Grimshaw et al. 2004, 2006, Eccles et al. 2005, Foy et al. 2005, Jamtvedt et al. 2006, Alexander \& Hearld 2009, Ivers et al. 2012, Sales et al. 2012). The sub-set of studies that have been conducted in the LTC context have a number of important limitations, leading to gaps in what is known about what works to improve LTC quality.

\section{Overview of the literature}

\section{Need for additional audit and feedback research in long-term care}

Audit and feedback is one quality improvement intervention that has been tested primarily among health care professionals in settings other than LTC, although some studies in LTC do exist. A review of randomized controlled trial (RCT) studies suggests that the effects of audit and feedback vary (Jamtvedt et al. 2006, Ivers et al. 2012). Some studies identified little or no effect while others have shown modest effects for improving both provider performance and patient outcomes (Jamtvedt et al. 2006, Ivers et al. 2012). There is also some evidence that this intervention is more effective - at least initially - in settings like LTC that have had little previous exposure to audit with feedback (Jamtvedt et al. 2006, Kalisch et al. 2007, Ivers et al. 2012) and when delivered with higher intensity (Jamtvedt et al. 2006, Ivers et al. 2012). Yet, previous audit with feedback studies conducted in LTC settings using a randomized trial design found little to no significant effects on care processes and patient outcomes (Rantz et al. 2001, Colon-Emeric et al. 2007).

Other studies using a variety of non-RCT designs have identified one or more improvements in care quality using both care process (e.g. assessment practice, prevention programmes) and patient outcome 
measures (e.g. pain, discomfort, falls) following a multifaceted intervention that included an audit with feedback component (Keay et al. 2003, Baier et al. 2004, Gama et al. 2011). However, these studies did not use a factorial design, and so could not parcel out the individual effects of the audit with feedback component (Keay et al. 2003, Baier et al. 2004, Gama et al. 2011).

Another limitation of this body of work is that, in the majority of studies (including the RCTs), high-level administrators or directors of care received the feedback reports, and purposeful targeting of front-line care providers did not occur. In these studies, the extent to which the feedback information filtered down to the level of front-line regulated staff members is unknown (Rantz et al. 2001, Keay et al. 2003, Baier et al. 2004, Colon-Emeric et al. 2007, Meijers et al. 2009, Gama et al. 2011). It is reasonable to expect that the use of the feedback information in practice, and subsequent effects on resident outcomes, may be affected according to who has accessed the information. If front-line care providers were to receive feedback reports, LTC administrators and the general public would likely expect them to enact changes to resident care based on the report information. However, the literature does not identify if this form of information is actually useful to front-line providers for making changes to care, or whether these providers actually received the report information in past studies.

\section{Opportunity for research in the current long-term care context}

The standardized resident data that are now available in LTC settings provides an additional impetus for conducting audit with feedback research in LTC. Within the Donabedian framework, the availability of structures such as standardized assessment tools (and the ability to derive quality measures from their data) may not improve resident outcomes when used alone. Appropriate processes also need to be in place to link these data to quality improvement practices, so positive effects on LTC resident outcomes can occur (Donabedian 2005). Long-term care facilities are an attractive environment to conduct audit with feedback interventions because they have standardized RAI data readily accessible for monitoring purposes. Yet, a literature review shows that RAI data have been underutilized in quality improvement studies in LTC settings (Sales et al. 2012). This study capitalizes on the existing RAI data, by using RAI data as the source for an audit with feedback intervention.

\section{Aim of study}

This is a sub-study of the Data for Improvement and Clinical Excellence (DICE) project, and will describe (primarily) front-line regulated care provider perceptions of a feedback report in LTC settings. Our main interest was to explore whether these care providers thought the feedback reports had information they could use to change resident care. The self-reported perceptions of this and the factors associated with those perceptions were examined.

The overall DICE study is described in detail in the previously published study protocol (Sales \& Schalm 2010). The larger study uses an interrupted time series design to test the effects of the audit with feedback intervention on resident outcomes.

\section{Methods}

\section{Design}

A cross-sectional survey design was used for this particular sub-study. The data represent a pooling of responses from three consecutive survey periods towards the end of the 13-month intervention.

\section{Intervention and sample}

Monthly feedback reports were prepared and hand delivered to providers working in four LTC facilities in Edmonton, Alberta, Canada, in 2009-10. The report depicted - using a simple line graph and some brief explanatory bullet points - the proportion of residents in their unit who had experienced pain, were at risk of a fall, had fallen, or had symptoms of depression in the most recent assessment period. Another line on the graph was included to indicate the proportions of residents in the eight other units who experienced a problem in each of these areas to provide a benchmark. Pain, falls, fall risk and depression were each represented on a separate graph. All four graphs were printed in colour and contained on one doublesided piece of paper. The bullets under each graph provided a brief explanation of the items used to create the graph and how the participant's unit compared with the other units in the study for each of the clinical care areas. Details on the report development and an example of the feedback report can be found in the study protocol paper (Sales \& Schalm 2010). The respondents were expected to find the report useful because it showed how their unit compared with other units on several clinical care areas that are important 
to quality of resident care, highlighting areas where the unit was doing well compared with the other units, and identifying areas for potential improvement.

Following feedback report distribution, surveys were administered to a convenience sample of providers at each of the sites. To be included in the study, providers simply needed to be working in one of the included facilities and could be either regulated or unregulated caregivers (health care aides). The perceptions of the unregulated caregivers was reported in a separate article (Fraser et al. 2013). The convenience sample of regulated caregivers included care managers (CMs), registered nurses (RNs), licensed practical nurses (LPNs), and a group of allied health professionals (AHPs) consisting of dieticians, social workers and pharmacists.

\section{Data collection}

Data were collected from respondents using a paperbased postfeedback survey. The survey was developed by the research team to gather respondent perceptions of the feedback reports around areas that could affect subsequent utilization of the feedback report information for the purposes of practice change (Sales \& Schalm 2010). A manual for drafting surveys based on the theory of planned behaviour was used to develop items (Ajzen 1991, Francis et al. 2004). The full content of the survey was published as an additional file in the protocol paper (Sales \& Schalm 2010). This sub-study focused on the survey items relating to respondent perceptions of the feedback reports. Surveys were administered on site on at least two days of the week, on day and evening shifts. Research assistants left additional reports and surveys for providers who were not on shift during their visit. Surveys were administered monthly, except for during regular holiday seasons, which are often times of low staffing (that is, July, August, December and January).

\section{Variables}

- Usefulness of feedback report information to make changes in resident care This question measured whether the respondent thought that the report was useful for the specific purpose of making changes to resident care (response options: yes or no). This was the dependent variable in the regression analysis.

- Types of changes If the respondent indicated the report was useful for making changes to resident care, he or she was asked to select one or more changes that they would like to make. Respondents were instructed to select all applicable options (response options: change the way residents are assessed; change the way residents are assisted in their activities of daily living; change the daily schedule for residents; change activities available for residents; change policies that affect residents or resident care; other kinds of change).

- Understood more than half of report This question measured how well the respondent understood the information in the report (response options: understood less than half, about half, more than half, all of it).

- Report useful or very useful (generally) This question measured whether the respondent thought that the report was useful, in general (response options: not useful, somewhat useful, useful, very useful).

- Interested in other data This question measured whether the report sparked interest in other types of data (response options: yes or no).

- Discussed the report with another staff member This question measured whether the provider discussed the report with co-workers (response options: yes or no).

Variables three to six were the independent variables in the analysis.

\section{Analysis}

All analysis was conducted using Stata ${ }^{\circledR} 10.0$ Statistical Software (StataCorp 2007, College Station, TX, USA). To increase statistical power, data were pooled from the three final survey periods. Because staff were asked to participate in surveys each month, staff members who participated in more than one survey during the three pooled survey periods were removed. The data collected were anonymized, so demographic information was used to link surveys that appeared to be the same person responding over more than one month. The calculated intra-class correlation coefficient $(\mathrm{ICC}=0.6)$ indicated a substantial correlation across the linked surveys. As a result, all but the first survey completed by the linked respondents were excluded.

For the multivariable logistic regression, the roles of different providers within the LTC context and the numbers of respondents available for the analysis were both considered. As registered nurses and licensed practical nurses are both regulated nursing providers involved in the front-line 24-hour care of LTC residents, these providers were grouped together in the initial model. In contrast to the front-line regulated 
nursing providers, allied health professionals have various responsibilities within the LTC context, and overall are involved in more episodic care than are regulated nurses who are present around-the-clock. While care managers are most often registered nurses, they have primarily an administrative or managerial role and the majority of their activities do not include direct resident care. Thus, the CM group was conceptualized as distinct from the RN group (who are RNs not in a CM role). Neither the AHP nor the CM groups contained sufficient numbers to conduct a logistic regression. As a result, logistic regression was used for the RN/LPN group only.

For the RN/LPN group, cross-tabulations and chisquare tests were computed between each independent variable and the dependent variable ('report provided useful information to make changes in resident care'). All variables tested in the bivariate analyses (whether significant or not) were included in the multivariable logistic regression analysis. Thus, the association of each independent variable with the dependent variable included adjustment for all variables of interest, regardless of their statistical significance in the multivariable analysis. A cluster correction was used to adjust for the variability introduced at the level of the nursing unit. Tests of significance were completed using the Wald Chi-squared test statistic at the 5\% level of significance.

For the logistic regression in the RN/LPN group, a provider group variable to adjust for RN or LPN status was included in the initial model. Both interaction of this variable with all other independent variables in the model, and potential confounding effects were assessed. There were no significant interaction terms and less than $15 \%$ change in all coefficients when the provider group variable was removed from the model. With insufficient evidence to suggest that either interaction or confounding were of concern for the provider group variable, the final model did not include a variable adjusting for provider group status.

\section{Results}

\section{Description of sample}

One hundred and twenty-six individual regulated care providers completed the post-feedback survey with valid responses to the study variables. The respondents had worked an average of 11.9 years in LTC facilities (SD 9.2; range 0.25-35) and an average of 5.4 years in the current unit (SD 5.3; range 0.17-26).

\section{Perceptions of feedback reports by provider group}

Overall, the majority of regulated providers reported that they understood more than half the content of the report $(78 \%)$ and that the report contained information useful for making changes to resident care $(64 \%)$. Slightly less than half the respondents $(39 \%)$ discussed the report with another staff member and $56 \%$ expressed interest in other forms of data (see Table 1).

While differences between groups were not tested for statistical significance due to a high probability of Type 1 error, there is some evidence suggesting that the different regulated provider groups held different perceptions of the feedback reports. The sample of AHPs reported less positive assessments of the feedback reports compared with the other provider groups. Of this group, 64\% reported understanding more than half the report, yet only $45 \%$ agreed that the report provided information useful for making changes to resident care. While all provider groups reported a high level of understanding (from 64$100 \%)$, care managers had some of the most positive responses to the other items. Licensed practical nurses responded more positively than RNs on all items, except for understanding the reports.

Of the providers who reported that the feedback information was useful for making changes to resident care, the majority of respondents specified that they

Table 1

Proportion of regulated care providers with a positive response to each variable

\begin{tabular}{|c|c|c|c|c|c|}
\hline Study variable & \multicolumn{5}{|c|}{ Regulated care provider group no. (\%) } \\
\hline Understood more than half of the report & $45(73.77)$ & $39(82.98)$ & $7(100.00)$ & $7(63.64)$ & $98(77.78)$ \\
\hline Report useful or very useful (generally) & $48(78.69)$ & $27(57.45)$ & $5(71.43)$ & $5(45.45)$ & $85(67.46)$ \\
\hline Discussed the report with another staff member & $24(39.34)$ & $17(36.17)$ & $5(71.43)$ & $3(27.27)$ & $49(38.89)$ \\
\hline
\end{tabular}

LPN, licensed practical nurse; RN, registered nurse; $\mathrm{CM}$, care manager; $\mathrm{AHP}$, allied health provider. 
Table 2

Proportion of regulated care providers with a positive response to each type of change to resident care

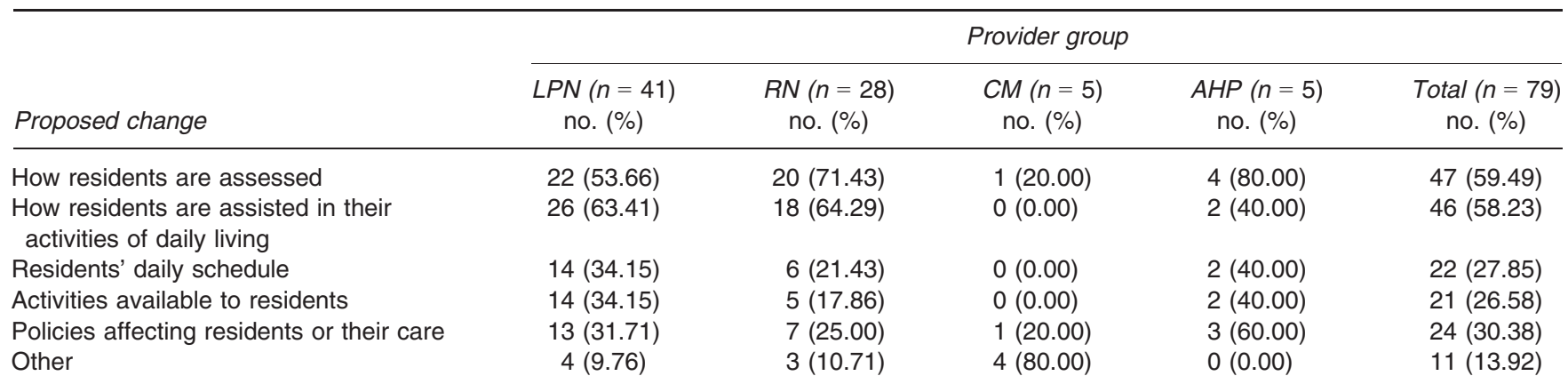

LPN, licensed practical nurse; RN, registered nurse; $\mathrm{CM}$, care manager; $\mathrm{AHP}$, allied health provider.

Table 3

Bivariate analyses: chi-square tests among regulated nurses $(n=108)$

\begin{tabular}{lccc}
\hline & \multicolumn{2}{c}{$\begin{array}{c}\text { Report gave useful information to } \\
\text { make changes in resident care }\end{array}$} \\
\cline { 2 - 4 } $\begin{array}{l}\text { So no. (\%) } \\
\text { Study variable }\end{array}$ & Yes no. (\%) & P value \\
\hline $\begin{array}{c}\text { Understood more } \\
\text { than half of report }\end{array}$ & $31(36.90)$ & $53(63.10)$ & 0.278 \\
$\begin{array}{c}\text { Report useful or } \\
\text { very useful (generally) }\end{array}$ & $15(20.00)$ & $60(80.00)$ & $<0.0001$ \\
$\begin{array}{c}\text { Discussed the report } \\
\text { with another staff member } \\
\text { Interested in other data }\end{array}$ & $11(26.83)$ & $30(73.17)$ & 0.203 \\
\hline
\end{tabular}

$P$ value using a chi-square test at the $5 \%$ level of significance.

would like to make changes to how residents are assessed (59\%) and to how residents are assisted in activities of daily living (58\%). Changes to resident schedules $(28 \%)$, activities $(27 \%)$ or policies $(30 \%)$ were less commonly identified (see Table 2).

\section{Factors associated with usefulness for making changes to resident care}

In the bivariate analysis, the dependent variable 'usefulness of information to make changes in resident care' was significantly associated only with stating that the report was generally useful $(P<0.0001)$ and with reporting an interest in other forms of data $(P<0.0001)$ (see Table 3). Neither understanding more than half the report nor discussing the report with other staff was significantly associated with finding the report useful for making changes to resident care. In multivariable logistic regression, statistically significant independent variables included both understanding more than half the report $(\mathrm{OR}=0.094,95 \%$ CI $0.015-0.60, P=0.013$ ) and perceiving the report as generally useful $(\mathrm{OR}=17.08,95 \%$ CI $5.14-56.74$,
Table 4

Multivariable logistic regression: associations with usefulness of feedback report information to make changes in resident care among regulated nurses $(n=108)$

\begin{tabular}{lccccc}
\hline Study variable & OR & $S E$ & $z$ & $P$ value & $95 \% \mathrm{Cl}$ \\
\hline $\begin{array}{c}\text { Understood } \\
\text { more than half } \\
\text { of report }\end{array}$ & 0.094 & 0.089 & -2.50 & 0.013 & $0.015-0.60$ \\
$\begin{array}{c}\text { Report useful or } \\
\text { very useful } \\
\text { (generally) }\end{array}$ & 17.08 & 10.46 & 4.63 & 0.000 & $5.14-56.74$ \\
$\begin{array}{c}\text { Discussed the } \\
\text { report with } \\
\text { another staff } \\
\text { member }\end{array}$ & 2.61 & 2.01 & 1.25 & 0.21 & $0.58-11.82$ \\
$\begin{array}{l}\text { Interested in } \\
\text { other data }\end{array}$ & 2.98 & 2.32 & 1.41 & 0.16 & $0.65-13.65$ \\
\hline
\end{tabular}

All variables were retained in the model, regardless of level of significance.

$P=0.000$ ) (see Table 4). Neither discussing the report with other staff nor expressing interest in other forms of data was statistically significant in the logistic regression analysis.

\section{Discussion}

The majority of front-line regulated care providers reported a high level of understanding of the audit with feedback reports. The regulated nursing staff, in particular, found the report information useful for making changes to resident care.

\section{Different roles may affect motivation to change care}

Regulated nurses (especially registered nurses) are expected to engage in a high level of leadership (McGilton et al. 2007, 2009), because in long-term 
care, the majority of front-line care providers are unregulated providers with limited training and education. Licensed practical nurses also hold supervisory roles within LTC settings (Schirm et al. 2000), so their positive perception of the feedback reports is not surprising. Given the expectations for leadership placed upon RNs, the trend towards LPNs responding more positively than RNs to the unit-level feedback information is somewhat unexpected. Yet, this finding is in keeping with other research comparing $\mathrm{RN}$ and LPN perceptions of their practice environments (Sales et al. 2005). Quality improvement efforts require substantial effort from leaders in these organisations, and if RNs hold a less positive view of the report information, this could prevent them from changing care in areas reported as problematic (Popejoy et al. 2000, Grando et al. 2007).

The allied health professionals had the least positive perceptions of the feedback reports. This may be explained by the nature of their role within Canadian LTC settings. In general, the work of AHPs is not unit-specific in LTC. They serve residents on multiple units within the LTC facility. Thus, they may have faced challenges when trying to determine how to interpret and improve unit-level resident outcomes (that is, outcomes calculated based on aggregated data from residents who live within a particular unit of a LTC facility). Feedback reports comparing only the residents that a particular group of AHPs serve with residents served by AHPs in other facilities may have been more meaningful to this group. In some ways, the AHP responses may reflect a barrier to interdisciplinary care providers working together to improve care for groups of residents. Recently, the importance of enhancing teamwork among professional nurses and unregulated caregiving staff has received increasing amounts of attention in the literature (Yeatts et al. 2004, Kalisch et al. 2007, Bishop et al. 2009). However, ways to enhance teamwork among regulated nurses and the AHPs who work on their units is an area in need of further research.

\section{Unexpected findings}

Understanding the report was expected to be essential in order to perceive the report as useful. However, our results indicate that those who understood more than half the report were less likely to indicate that it provided useful information for making changes to resident care. This unusual finding will be explored in more detail in several follow-up focus groups in two of the LTC facilities in the study, to gain a more in-depth understanding into which aspects of the report were understandable, the specific meaning of usefulness to the regulated nurses, and their perceptions of the meaning of a significant negative relationship between these variables.

One potential explanation for this unexpected finding is the individualistic nature of the practice change survey question. The particular survey question asked whether the respondent found the report useful for making changes to their own practice. Of interest, when respondents who indicated that they would like to change their practice were asked to identify the areas of practice that they would like to change, the most common responses were individualistic types of changes: assessment practice (which RNs and LPNs are responsible for every shift) and assisting residents in activities of daily living (the most common delegated activity in LTC, which occurs under the supervision of the RNs and LPNs). In contrast, the changes that were identified less often related more to organisational schedules, activities and policies, which the RNs may feel that they have less control over.

However, the feedback reports do not identify only those client outcomes for a specific regulated provider's client load, they are a measure of how a nursing unit functions. It may be that those who reported understanding more than half of the feedback report also had a better understanding of the organisational nature of the measures on the report. It is possible that the more one understands about how unit-level quality indicator scores are constructed, and what might be required to change them, the less likely one is to state that this information is useful to inform their own practice if one believes that they do not have the power to change organisational practices. Despite the fact that regulated providers are in formal leadership positions in LTC, this explanation would be in keeping with research which found that regulated nurses in LTC pay little attention to their leadership role in their daily practice (McGilton et al. 2009).

Lastly, it is interesting that discussing the unit-level feedback information with other colleagues did not appear to affect the extent to which the RN/LPN provider group felt they could improve resident care. This suggests that the regulated nurse providers felt they could make improvements to care based on the information in the feedback report, regardless of whether they have discussed the information with their colleagues. This is an interesting finding in a context in which regulated providers deliver only a small proportion of direct resident care. Regulated providers in this 
sample did not appear to feel the need to discuss the information from the feedback report with other care providers - notably the unregulated caregivers - to make changes to resident care.

\section{Limitations}

None of the facilities enrolled in this study had a reliable and standardized process for documenting how many employees worked for them at any given time. This led to substantial challenges in determining response rates for this study, because the number of providers working on a particular shift was unknown. Response rates, based on the number of surveys distributed compared with the number returned, in the final three survey cycles ranged from 49.9 to $83.4 \%$. However, this number does not differentiate response rates by provider group; rather, it is the response rate for all respondents in the larger study, including both regulated and unregulated providers. The particular response rate for the regulated providers described in this article is not reported, because only the provider status of people who completed the surveys (and not of those who were asked but chose not to complete the survey) is known.

For each of the RN, LPN, CM and AHP groups, proportions were calculated for categorical variables and means for continuous variables. Comparisons among provider groups are descriptive only, as testing for differences between the four provider groups on multiple variables would have led to a high chance of a false-positive result (that is, a Type I error - detecting a significant difference between the groups by chance when none exists in reality).

Generalization of these findings is limited by the small sample size of the care manager and allied health professional provider groups. There are only a few care managers working in a given facility, so the small care manager sample size was not an issue with response rate (as each unit typically has only one care manager). The AHP group is small in number and represents a group of individuals who - while more similar to one another than to the regulated nurse providers - comprises a variety of professionals who have different roles within the LTC setting. Thus, the aggregated view of this group may not accurately represent the different views of the social workers, dieticians and pharmacists who comprise the AHP group. A much larger sample of facilities is needed to gain responses that are more representative of both the $\mathrm{CM}$ and AHP groups, and for the individual professional groups comprising the larger AHP group.
The use of self-report is a further limitation. This may be particularly problematic for evaluating understanding of the reports, as measuring perceptions of understanding may not be equivalent to objectively evaluating a respondent's understanding (in other words, respondents may inaccurately perceive how much they know). However, for the purpose of the logistic regression analysis, it is reasonable to expect perceptions of understanding to be related to perceptions of usefulness to making changes to resident care.

\section{Conclusions}

There is a need to increase care quality in nursing homes. One quality improvement approach uses existing RAI data to develop feedback reports. These reports compare residents in different long-term care units on important areas related to care quality. Care providers can then use this information as a stimulus to change resident care practices. This study suggests that front-line care providers hold positive perceptions of receiving feedback report information, and find the information understandable and useful for making changes to resident care.

\section{Implications for nursing management}

The findings on the perceptions of understanding and usefulness of the feedback report information among front-line care providers supports a more collaborative approach to quality improvement in long-term care. In another study designed to identify perceptions held by site staff regarding an audit with feedback intervention, administrators did not agree on who should have access to the feedback report information (Grando et al. 2007). Some believed they should share the reports with all staff, others wanted access limited to the quality improvement team, and still others wanted access restricted to administrators only (Grando et al. 2007). Our findings suggest that some of these administrators' assumptions are not in line with the information needs and preferences of direct care providers. Our finding that discussions with others was not associated with stating that the report contained useful information to change practice suggests that a lack of communication about quality may also exist among non-managerial RNs and LPNs (and not just between management and front-line providers). Another study identified that ensuring communication with and active participation of front-line care providers was essential for improving quality within LTC settings 
(Rantz et al. 2012). Taken together, this work suggests that discussions about quality among and between facility/unit managers and RNs/LPNs in LTC should become the norm of managerial and front-line nursing practice, and that focused attention will be required to institute this norm.

Reporting that one wants to change care practice and actually making changes to resident care are not equivalent; future analysis will determine whether the DICE audit with feedback intervention, targeted to front-line staff, had a significant effect on resident outcomes. However, almost all (79 of 81 ) providers who said they found the report useful for making changes to resident care also answered the question on how care should be improved. This is an indication that they were being thoughtful about answering the question, and may actually implement the changes they suggested.

Nursing managers, including LTC administrators and unit-level managers, are encouraged to examine their own assumptions around the information needs of the regulated front-line care providers who they provide leadership to in their settings. Consideration should be given to how unit-level information on care quality can be shared with regulated care providers across all levels of the LTC staffing hierarchy, as a first step to working collaboratively towards delivering high quality resident care.

\section{Acknowledgements}

We gratefully acknowledge the intellectual input from the full research team for this project: Marian Anderson, Tracey Bucknall, Thorsten Duebel, Kari Elliott, Carole Estabrooks, Gloria Gao, Manal Kleib, Vivien Lai, Lili Liu, Suzanne Maisey, Anastasia Mallidou, Lynne Mansell, Colleen Maxwell, Iris Neumann, Corinne Schalm and Sharon Warren.

\section{Source of funding}

Funding for this work was provided by the Alberta Heritage Foundation for Medical Research (CA\#3235) and the Canadian Health Services Research Foundation (RC2 1781).

\section{Ethical approval}

The DICE project received ethics approval from the Health Research Ethics Board at the investigators' local university (Pro00003694), and operational approval from the participating LTC organizations.

\section{References}

Ajzen I. (1991) The theory of planned behavior. Organizational Behavior and Human Decision Processes 50, 179-211.

Alberta Office of the Auditor General. (2005) Report of the Auditor General on Seniors Care and Programs. Available at: http:// www.oag.ab.ca/files/oag/OAG_Seniors_2005.pdf, accessed 28 March 2012.

Alexander J.A. \& Hearld L.R. (2009) What can we learn from quality improvement research? A critical review of research methods Medical Care Research and Review 66 (3), 235-271.

Baier R.R., Gifford D.R., Patry G. et al. (2004) Ameliorating pain in nursing homes: a collaborative quality-improvement project. Journal of the American Geriatrics Society 52 (12), 1988-1995.

Berta W., Laporte A., Zarnett D., Valdmanis V. \& Anderson G. (2006) A pan-Canadian perspective on institutional long-term care. Health Policy, 79 (2-3), 175-194.

Berwick D.M. (2008) The science of improvement. Journal of the American Medical Association 299 (10), 1182-1184.

Bishop C.E., Squillace M.R., Meagher J., Anderson W.L. \& Wiener J.M. (2009) Nursing home work practices and nursing assistants' job satisfaction. The Gerontologist 49 (5), 611-622.

Bostrom A.M., Slaughter S.E., Chojecki D. \& Estabrooks C.A. (2012) What do we know about knowledge translation in the care of older adults? A scoping review. Journal of the American Medical Directors Association 13 (3), 210-219.

Colon-Emeric C.S., Lyles K.W., House P. et al. (2007) Randomized trial to improve fracture prevention in nursing home residents. American Journal of Medicine 120 (10), 886-892.

Donabedian A. (2005) Evaluating the quality of medical care... reprinted from the Milbank Memorial Fund Quarterly, vol. 44, no. 3, pt. 2, 1966 (pp. 166-203). Milbank Quarterly 83 (4), 691-729.

Eccles M., Grimshaw J., Walker A., Johnston M. \& Pitts N. (2005) Changing the behavior of healthcare professionals: the use of theory in promoting the uptake of research findings. Journal of Clinical Epidemiology 58 (2), 107-112.

Foy R., Eccles M.P., Jamtvedt G., Young J., Grimshaw J.M. \& Baker R. (2005) What do we know about how to do audit and feedback? Pitfalls in applying evidence from a systematic review BMC Health Services Research 13 (5), 50.

Francis J.J., Eccles M., Johnston M. et al. (2004) Constructing Questionnaires Based on the Theory of Planned Behaviour: A Manual for Health Services Researchers. Center for Health Services Research University of Newcastle, Newcastle upon Tyne.

Fraser K.D., O’Rourke H.M., Baylon M.A.B., Boström A.M. \& Sales A.E. (2013) Unregulated provider perceptions of audit and feedback reports in long-term care: cross-sectional survey findings from a quality improvement intervention. BMC Geriatrics 13, doi: 10.1186/1471-2318-13-15.

Gama Z.A., Medina-Mirapeix F. \& Saturno P.J. (2011) Ensuring evidence-based practices for falls prevention in a nursing home setting. Journal of the American Medical Directors Association 12 (6), 398-402.

Grando V.T., Rantz M.J. \& Maas M. (2007) Nursing home staff's views on quality improvement interventions: a followup study. Journal of Gerontological Nursing 33 (1), 40-47. 
Grimshaw J., Eccles M. \& Tetroe J. (2004) Implementing clinical guidelines: current evidence and future implications. Journal of Continuing Education in the Health Professions 24 (Suppl 1), S31-S37.

Grimshaw J., Eccles M., Thomas R. et al. (2006) Toward evidence-based quality improvement - evidence (and its limitations) of the effectiveness of guideline dissemination and implementation strategies 1966-1998. Journal of General Internal Medicine 21, S14-S20.

Hawes C., Morris J.N., Phillips C.D., Mor V., Fries B.E. \& Nonemaker S. (1995) Reliability estimates for the minimum data set for nursing home resident assessment and care screening (MDS). The Gerontologist 35 (2), 172-178.

Hirdes J.P., Mitchell L., Maxwell C.J. \& White N. (2011) Beyond the 'iron lungs of gerontology': using evidence to shape the future of nursing homes in Canada. Canadian Journal on Aging 30 (3), 371-390.

Institute of Medicine. (1986) Improving the Quality of Care in Nursing Homes. National Academy Press, Washington, DC.

Institute of Medicine. (2001) Improving the Quality of LongTerm Care. National Academic Press, Washington, DC.

Ivers N., Jamtvedt G., Flottorp S. et al. (2012) Audit and feedback: effects on professional practice and healthcare outcomes. Cochrane Database Systematic Reviews 6, CD000259.

Jamtvedt G., Young J.M., Kristoffersen D.T., O’Brien M.A. \& Oxman A.D. (2006) Audit and feedback: effects on professional practice and health care outcomes. Cochrane Database of Systematic Reviews 2, CD000259.

Kalisch B.J., Curley M. \& Stefanov S. (2007) An intervention to enhance nursing staff teamwork and engagement. Journal of Nursing Administration 37 (2), 77-84.

Keay T.J., Alexander C., McNally K., Crusse E. \& Eger R.E. (2003) Nursing home physician educational intervention improves end-of-life outcomes. Journal of Palliative Medicine 6 (2), 205-213.

McDaniel S.A. (2009) The conundrum of demographic aging and policy challenges: a comparative case study of Canada, Japan and Korea. Canadian Studies in Population, 36 (1-2), 37-62.

McGilton K.S., McGillis Hall L., Boscart V. \& Brown M. (2007) Effects of director of care support on job stress and job satisfaction among long-term care nurse supervisors. Nursing leadership 20 (3), 52-66.

McGilton K.S., Bowers B., McKenzie-Green B., Boscart V. \& Brown M. (2009) How do charge nurses view their roles in longterm care? Journal of Applied Gerontology 28 (6), 723-742.

Meijers J.M., Candel M.J., Schols J.M., Schueren M. \& Halfens R.J. (2009) Decreasing trends in malnutrition prevalence rates explained by regular audits and feedback. Journal of Nutrition 139 (7), 1381-1386.

Popejoy L.L., Rantz M.J., Conn V., Wipke-Tevis D., Grando V.T. \& Porter R. (2000) Improving quality of care in nursing facilities: gerontological clinical nurse specialist as research nurse consultant. Journal of Gerontological Nursing 26 (4), 6-13.

Rantz M.J., Popejoy L., Petroski G.F. et al. (2001) Randomized clinical trial of a quality improvement intervention in nursing homes. The Gerontologist 41 (4), 525-538.
Rantz M.J., Zwygart-Stauffacher M., Flesner M. et al. (2012) Challenges of using quality improvement methods in nursing homes that 'need improvement'. Journal of the American Medical Directors Association 13 (8), 732-738.

Report of the Auditor General of Alberta. (2008) Systems-health and Wellness/Seniors and Community Supports. 95-147. Available at: http://www.continuingcarewatch.com/pdf/ Auditor\%20General\%20Report \%20on \%20Seniors \%20Care $\% 20$ and $\% 20$ Program s\%20April\%202008.pdf, accessed 2 March 2012.

Saher M.N. (2011) Report of the Auditor General of Alberta. Available at: http://www.oag.ab.ca/files/oag/OAGNov2011 report.pdf, accessed 2 March 2012.

Sales A.E. \& Schalm C. (2010) Data for improvement and clinical excellence: protocol for an audit with feedback intervention in long-term care. Implementation Science 5 (74), 1-7.

Sales A.E., Sharp N.D., Li Y. et al. (2005) Nurse staffing and patient outcomes in Veterans Affairs hospitals. Journal of Nursing Administration 35 (10), 459-466.

Sales A.E., Bostrom A.M., Bucknall T. et al. (2012) The use of data for process and quality improvement in long-term care and home care: a systematic review of the literature. Journal of the American Medical Directors Association 13 (2), 103-113.

Schirm V., Albanese T., Garland T.N., Gipson G. \& Blackmon D.J. (2000) Caregiving in nursing homes: views of licensed nurses and nursing assistants. Clinical Nursing Research 9 (3), 280-297.

Shojania K.G. \& Grimshaw J.M. (2005) Evidence-based quality improvement: the state of the science. Health Affairs 24 (1), 138-150.

StataCorp (2007) Stata ${ }^{\circledR}$ Statistical Software: Release 10. StataCorp LP, College Station, TX, USA.

Statistics Canada. (2007) A Portrait of Seniors in Canada 2006 (No. 89-519-XIE). Available at: http://www.statcan.gc.ca/ pub/89-519-x/89-519-x2006001-eng.pdf, accessed 2 March 2012.

Statistics Canada. (2008) Canadian Demographics at a Glance (No. 91-003-X). Ottawa, ON: Minister of Industry. Available at: www.statcan.ca, accessed 2 March 2012.

Swafford K.L., Miller L.L., Tsai P., Herr K.A. \& Ersek M. (2009) Improving the process of pain care in nursing homes: a literature synthesis. Journal of the American Geriatrics Society 57 (6), 1080-1087.

Wiener J.M. (2003) An assessment of strategies for improving quality of care in nursing homes. The Gerontologist, 43 (Spec no 2), 19-27.

Yeatts D.E., Cready C., Ray B., DeWitt A. \& Queen C. (2004) Self-managed work teams in nursing homes: implementing and empowering nurse aide teams. The Gerontologist 44 (2), 256-261.

Zimmerman D.R. (2003) Improving nursing home quality of care through outcomes data: the MDS quality indicators. International Journal of Geriatric Psychiatry 18 (3), 250257. 